Original Research Paper

\title{
Characterization, Antioxidant Activities and Optimization of Preparation Process of a Auricularia Polytricha Polysaccharide-Zinc (II) Complex
}

\author{
Xinyi Wu, Xiao Ding, Ze Zhang, Ziyi Xu, Jiehan Zhang, Wen Zhao and *Yiyong Chen \\ School of Biology and Food Engineering, Changshu Institute of Technology, Changshu 215500, China
}

\author{
Article history \\ Received: 14-01-2021 \\ Revised: 07-03-2021 \\ Accepted: 12-03-2021 \\ Corresponding Author: \\ Yiyong Chen \\ School of Biology and Food \\ Engineering, Changshu \\ Institute of Technology, \\ Changshu 215500, China \\ Email: greenpop6688@126.com
}

\begin{abstract}
In our previous work, a novel polysaccharide APP3a from Auricularia polytricha was successfully isolated and purified. Response surface methodology was used to optimize the preparation process of APP $3 a-Z n$ on the basis of the single factor experiments. Characterization of APP3a-Zn was carried out and its antioxidant activity was also investigated. The result showed that the best preparation process of APP3a-Zn was chelation time $4.45 \mathrm{~h}$, chelation temperature $49.37^{\circ} \mathrm{C}$ and $\mathrm{pH} 4.68$. The weight loss of APP3a-Zn was slightly smaller than that of APP3a within the heating temperature range and the crystal structure of APP3a-Zn had undergone tremendous changes compared with APP3a. APP3a-Zn had stronger antioxidant activity than APP3a. The results indicated that chelation with zinc enhanced the antioxidant activity of APP3a. In the food industry, APP3a-Zn had great potential as a new zinc supplement with antioxidant activity.
\end{abstract}

Keywords: Auricularia polytricha, Zinc, Polysaccharide, Chelation, Characterization, Antioxidant Activities

\section{Introduction}

Zinc $(\mathrm{Zn})$ is one of the essential trace elements in living organisms, which plays an important role in the normal cell function and human metabolism (Beyersmann and Haase, 2001; Wang et al., 2019a). Recently, zinc deficiency can lead to developmental delays in children, which has become the main cause of public health (Caulfield et al., 2004a; 2004b) and a decline in learning and memory (Boroujeni et al., 2009). Furthermore, zinc deficiency can cause oxidative damage to the human body because of the inhibition of the formation of Reactive Oxygen Species (ROS) (Harmaza and Slobozhanina, 2014). At present, Zn supplement includes organic zinc (polysaccharide or amino acid chelate) and inorganic zinc (zinc sulfate or zinc oxide). Inorganic zinc is the most common. However, in the case of excessive intake, inorganic zinc has low absorption and toxicity to the human body. Because organic zinc is closer to the action form of zinc in the body, it has significant advantages in human utilization and physiological activity (Buff et al., 2005). Therefore, it is necessary to develop a safe, effective, long-term and non-toxic zinc supplement agent, which provides an alternative to improve the zinc content in human body.
The hydroxyl and/or amino functional groups in polysaccharides can form chelation with metals or some non-metals through chemical adsorption (Wang et al., 2019b). After chelating modification, polysaccharides can improve their own biological activity and have new functions (Zhao et al., 2017). The chelated metals are more easily absorbed by organisms (Wang and Betti, 2017). Some researchers have studied the metal-binding ability of polysaccharides and prepared metal-polysaccharide complexes or chelates successfully, such as seleniumpolysaccharides (Lian et al., 2018; Li et al., 2016). Complexation modification of polysaccharides has become one of the most important modification methods.

Auricularia polytricha belongs to Auriculariaceae family and is widely used as a food or tonic in China with some functions such as lowering blood-fat, antitumor and immunomodulatory activities (Sheu et al., 2004). Polysaccharides from A. Polytricha have been shown to have anti-cancer activity (Yu et al., 2014) and anti-hypercholesterolemia (Zhao et al., 2015). To the best of our knowledge, there is no report on optimization of preparation technology, characterization and biological activity of $A$. polytricha polysaccharideZinc(II) complex. 
In our previous work, a novel polysaccharide APP3a from Auricularia polytricha was successfully isolated and purified (Chen and Xue, 2018). However, there is no report on the chelation between APP3a and zinc (II) and its biological activity. Response Surface Method (RSM) can determine the best processing parameters effectively (Wang et al., 2016). Therefore, RSM was used to optimize the preparation process of APP3a-zinc (II) complex (APP3a-Zn). In addition, the characterization and antioxidant activity of APP3a-Zn were also studied. The present study aimed to explore the effect of chelation modification on the antioxidant activity of APP3a-Zn, determine the preparation process of APP3a-Zn and lay the foundation for the development of new biological zinc supplements.

\section{Materials and Methods}

\section{Materials and Chemicals}

A novel polysaccharide APP3a from Auricularia polytricha was successfully isolated and purified, which consisted of arabinose, mannose, glucose and galactose in a molar ratio of $1: 1.33: 1.06: 1.23$ with an average molecular weight of 21,242 $\mathrm{Da}$ as we presented in our previous work (Chen and Xue, 2018). Zn Standard solution (1000 $\mathrm{mg} / \mathrm{L})$ was purchased from national center for analysis and testing of nonferrous metals and electronic materials (Beijing, China); 1,1-Diphenyl-2Picrylhydrazyl (DPPH) was purchased from Sigma Aldrich (Sigma, St Louis, MO, USA); All other chemicals and solvents were of analytical reagent grade.

\section{Chelation Rate Determination}

The standard solutions with different gradient concentrations $(0,5.0,10.0,20.0,40.0$ and $60.0 \mathrm{mg} / \mathrm{L})$ were prepared from a $\mathrm{Zn}$ standard solution $(1000 \mathrm{mg} / \mathrm{L})$ by dilution with $\mathrm{HNO}_{3}$ solution (3.3\%). An atomic absorption spectrophotometer (Z-2000, Hitachi, Japan) was used to determine the absorbance. A standard curve of absorbance versus $\mathrm{Zn}$ content was obtained.

APP3a-Zn (100 mg) was digested in a digestion tank with a mixture of $\mathrm{HNO}_{3}$ and $\mathrm{HClO}_{4}(5 \%)$. Then, the digestion solution was diluted with water to a volume of $100 \mathrm{~mL}$. After filtration, atomic absorption spectrophotometry was used to measure the $\mathrm{Zn}$ content. Concentration of zinc was calculated from the standard curve. Equation 1 was used to calculate the chelation rate as follows:

$$
\text { Chelation rate }(\%)=C V / M * 100
$$

where, $C$ means concentration of zinc. $V$ means dilution volume $(100 \mathrm{~mL}) . M$ means the quantity of APP3a-Zn (100 mg).

\section{APP3a-Zn Preparation}

$25 \mathrm{~mL} \mathrm{ZnSO}_{4}$ solution $(0.45 \mathrm{~mol} / \mathrm{L})$ and $25 \mathrm{~mL}$ APP3a solution $(2.5 \mathrm{mg} / \mathrm{mL})$ were mixed thoroughly and evenly. The reaction condition including $\mathrm{pH}$, temperature and chelating time was set. After the chelation reaction was accomplished, the mixture was dialyzed using a dialysis membrane with a molecular weight cut-off of $2500 \mathrm{Da}$ ) for $10 \mathrm{~h}$, freeze-dried and finally APP3a-Zn was obtained.

\section{Experiment Design of RSM}

The ranges of the factors were determined based on preliminary single tests. The response value was chelation rate, a central composite design with three independent variables $\left(\mathrm{X}_{1}\right.$, Chelation time, $\mathrm{X}_{2}$, Chelation temperature and $\mathrm{X}_{3}, \mathrm{pH}$ ) and three levels was chosen to optimize the preparation technology conditions of APP3a-Zn.

\section{Determination of Zinc Content in APP3a-Zn}

APP3a was used as a control, determination of zinc content in APP3a-Zn was performed by flame atomic absorption spectrometric method. Briefly, $0.1 \mathrm{~g}$ APP3a-Zn and $0.1 \mathrm{~g}$ APP3a were mixed with $10 \mathrm{~mL}$ $\mathrm{HNO}_{3}$ and $2 \mathrm{~mL} \mathrm{HClO}_{4}$ and hydrolyzed at $160^{\circ} \mathrm{C}$ for 12 $\mathrm{h}$, respectively. After the hydrolysis was complete, the sample was diluted with $5 \mathrm{~mL}$ water and then analyzed by atomic absorption spectrophotometer (AA6100, Shimadzu Co., Japan).

\section{FT-IR Spectroscopy}

$1 \mathrm{mg}$ of APP3a-Zn and APP3a was weighed respectively, mixed with spectral grade potassium bromide after drying and then pressed into pellets. Using an FT-IR spectrophotometer (Nicolet 6700, Thermo Scientific, USA), the infrared spectra of APP3a-Zn and APP3a were analyzed at the scanning range of $400-4000 \mathrm{~cm}^{-1}$.

\section{$X$-Ray Diffraction Spectrum}

The X-ray diffraction spectrum of APP3a-Zn was determined by a X-ray diffractometer (SCXmini, Rigaku Inc., Tokyo, Japan). The operating parameters were set as follows: $\mathrm{Cu} \mathrm{K} \alpha$ radiation, $40 \mathrm{kV}, 200 \mathrm{~mA}$ and a scan rate of $0.5^{\circ} \mathrm{C} / \mathrm{min}$ under a nitrogen atmosphere.

\section{Thermogravimetric Analysis}

Thermogravimetric analysis (TGA) was carried out on a Thermo Gravimetric Analyzer (TGA/SDTA851, Mettler Toledo Instruments Co., Ltd., Switzerland). $1 \mathrm{mg}$ sample was inoculated into the sample pan. Thereafter, the sample was heated from 20 to $800^{\circ} \mathrm{C}$ at a heating rate of $10^{\circ} \mathrm{C} / \mathrm{min}$ under a nitrogen atmosphere. 


\section{Morphological Analysis}

The surface structure of APP3a-Zn and APP3a was investigated by Scanning Electron Microscope (SEM) (Ultra Plus, Zeiss, Germany). The sample was fixed on a clean glass slide with a double-sided carbon conductive adhesive tap. Morphological characterization of the powder was observed by an In-len secondary electron detector (GEMINI).

\section{Determination of Antioxidant Activity}

\section{Determination of DPPH Radical Scavenging Activity}

A $2 \mathrm{~mL}$ volume of APP3a or APP3a-Zn $(0.2,0.4$, $0.6,0.8,1.0 \mathrm{mg} / \mathrm{mL}$ ) was added into $1 \mathrm{~mL}$ of DPPH solution $(0.1 \mathrm{mM}$, in $95 \%$ ethanol). The mixture was shaken vigorously and allowed to stand at $30^{\circ} \mathrm{C}$ for 30 min in dark. The absorbance at $517 \mathrm{~nm}$ was measured. Distilled water instead of polysaccharide solution was used as a blank control. All measurements were performed in triplicate. Equation 2 was used to calculate DPPH radical scavenging rate as follows:

$$
\text { DPPH radical scavenging rate }(\%)=\left(1-A / A_{0}\right) \times 100
$$

where, $A$ represents the absorbance value of samples. $A_{0}$ represents the absorbance value of the DPPH solution without samples.

\section{Determination of Hydroxyl Radical Scavenging Activity}

A $1.0 \mathrm{~mL}$ volume of APP3a or APP3a-Zn $(0.2,0.4$, $0.6,0.8,1.0 \mathrm{mg} / \mathrm{mL}$ ) was added into $1.0 \mathrm{~mL}$ salicylic acid ethanolic solution $(9.0 \mathrm{mmol} / \mathrm{L})$ and $1.0 \mathrm{~mL} \mathrm{FeSO}_{4}$ solution $(9.0 \mathrm{mmol} / \mathrm{L})$. Then $1.0 \mathrm{~mL} \mathrm{H}_{2} \mathrm{O}_{2}(8.8 \mathrm{mmol} / \mathrm{L})$ was added into the mixture to start the reaction at $37^{\circ} \mathrm{C}$ for $30 \mathrm{~min}$. The absorbance at $510 \mathrm{~nm}$ was measured. Distilled water instead of polysaccharide solution was used as a blank control. All measurements were performed in triplicate. Equation 3 was used to calculate hydroxyl radical scavenging rate as follows:

$$
\begin{aligned}
& \text { Hydroxyl radical scavenging rate }(\%) \\
& =\left[1-\left(A_{2}-A_{1}\right) / A_{0}\right] \times 100
\end{aligned}
$$

where, $A_{0}$ represents the absorbance value of distilled water instead of samples. $A_{1}$ represents the absorbance value of distilled water instead of salicylic acid. $A_{2}$ represents the absorbance value of samples.

\section{Determination of Superoxide Anion Radical Scavenging Activity}

A $1.0 \mathrm{~mL}$ volume of APP3a or APP3a-Zn $(0.2,0.4$, $0.6,0.8,1.0 \mathrm{mg} / \mathrm{mL}$ ) was added into $4.5 \mathrm{~mL}$ Tris- $\mathrm{HCl}$ buffer solution $(50 \mathrm{mmol} / \mathrm{L}, \mathrm{pH} 8.2)$ and $3.2 \mathrm{~mL}$ distilled water at $25^{\circ} \mathrm{C}$ for $20 \mathrm{~min}$. Then $0.3 \mathrm{~mL}$ pyrogallol was added into the mixture to react at $25^{\circ} \mathrm{C}$ for $3 \mathrm{~min} .1 \mathrm{drop}$ of $10 \mathrm{~mol} / \mathrm{L} \mathrm{HCl}$ was added immediately to stop the reaction. The absorbance at $325 \mathrm{~nm}$ was measured. Distilled water instead of polysaccharide solution was used as a blank control. All measurements were performed in triplicate. Equation 4 was used to calculate superoxide anion radical scavenging rate as follows:

$$
\begin{aligned}
& \text { Superoxide anion radical scavenging rate }(\%) \\
& =\left(1-A / A_{0}\right) \times 100
\end{aligned}
$$

where, $A$ represents the absorbance value of samples. $A_{0}$ represents the absorbance value of distilled water.

\section{Statistical Analysis}

Experimental design and data analysis were performed by a Design Expert ${ }^{\circledR}$ software. The analysis of variance and significant differences were performed by Analysis of Variance (ANOVA) program. All values with $p<0.05$ were considered statistically significant.

\section{Results}

\section{Effect of Process Parameters on Chelation Rate}

Figure 1A showed the effect of chelation time on chelation rate. It could be seen that chelating rate increased continuously with chelating time when chelating time was less than $4 \mathrm{~h}$. When chelating time was $4 \mathrm{~h}$, chelation rate was the highest. When chelation time increased again, chelation rate decreased instead. The reason may be that the continuously increasing chelation time leads to the destruction of the stability of the reaction system. The increase in the production rate of side reaction resulted in the dissociation of the complex and the reduction of chelation (Wang et al., 2016). Therefore, the best chelating time of APP3a-Zn was $4 \mathrm{~h}$.

Figure 1B showed the effect of chelation temperature on chelation rate. It could be seen that chelation rate gradually increased with the temperature rising when temperature was lower than $50^{\circ} \mathrm{C}$. However, when the temperature exceeded $50^{\circ} \mathrm{C}$, chelation rate began to decline. It may be because that when the boundary temperature increased, it promoted APP3a to swell and allowed zinc to penetrate into the polysaccharide particles, thereby effectively increasing the collision and chelation rate between molecules (Imai et al., 2004). When temperature exceeded $50^{\circ} \mathrm{C}$, excessive temperature led to the degradation of APP3a easily and reduced the chelation rate. Therefore, the optimal chelating temperature was $40^{\circ} \mathrm{C}$. 

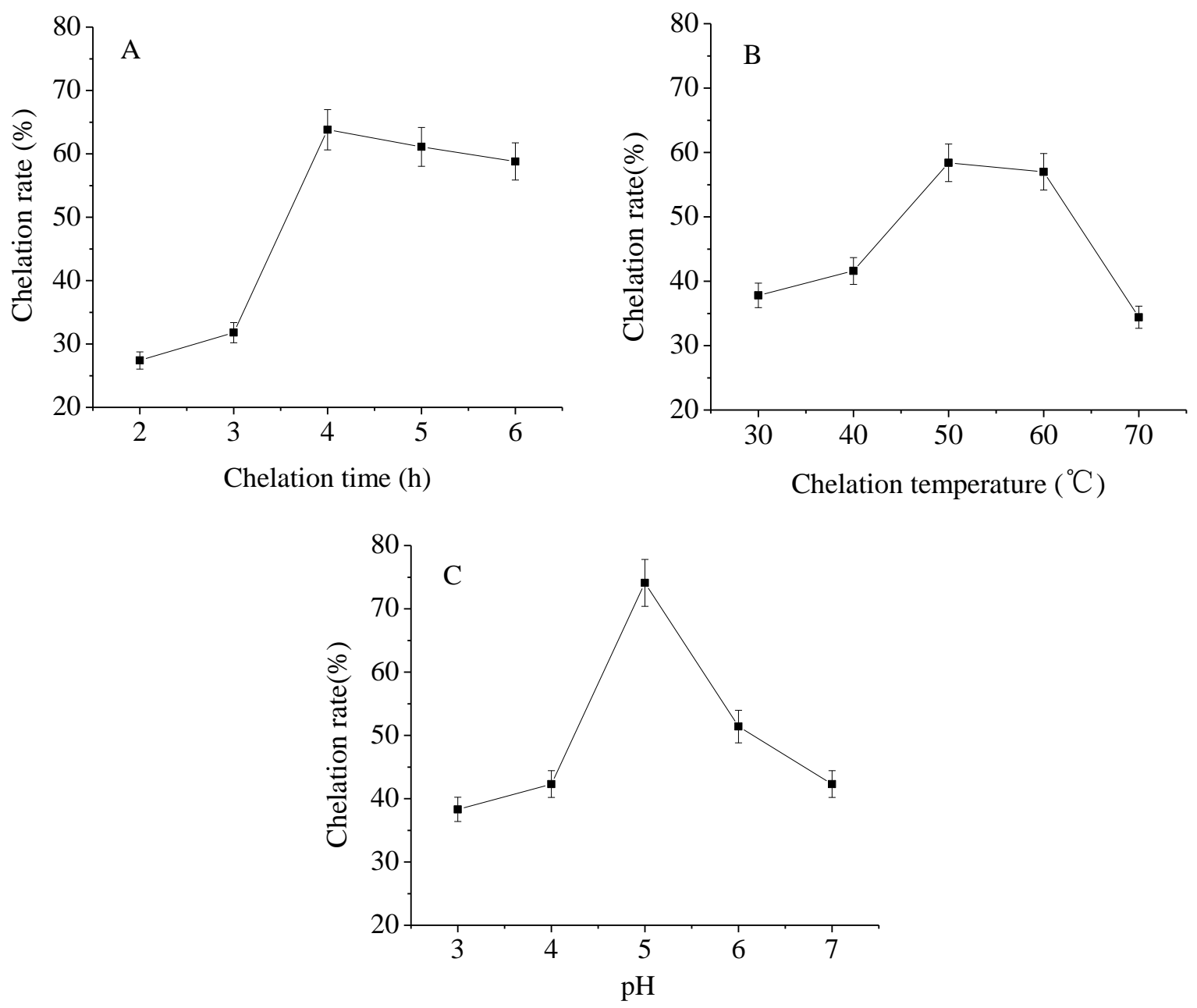

Fig. 1: Effect of chelation time (A), chelation temperature (B) and $\mathrm{pH}(\mathrm{C})$ on the chelation rate of APP3a-Zn

Figure 1C showed the effect of $\mathrm{pH}$ on chelation rate. When $\mathrm{pH}$ increased from 3 to 5 , chelation rate gradually increased. When $\mathrm{pH}$ was 5 , chelation rate was the highest. However, when $\mathrm{pH}$ exceeded 5, chelation rate began to decline. The reason may be that the increase of $\mathrm{pH}$ will produce $\mathrm{Zn}(\mathrm{OH})_{2}$ precipitation, so the concentration of $\mathrm{Zn}^{2+}$ reduces, which results in reduction of chelation rate. Therefore, the optimal chelating $\mathrm{pH}$ was 5.

\section{Statistical Analysis and the Model Building}

Based on single factor experiments, factors and levels designed for RSM were shown in Table 1. Result of response surface analysis of the variation of chelation rate of APP3a-Zn with chelation time $\left(X_{1}\right)$, chelation temperature $\left(X_{2}\right)$ and $\mathrm{pH}\left(X_{3}\right)$ was presented in Table 2. Chelation rate $(Y)$ was predicted by means of second order polynomial equation:

$$
\begin{aligned}
& Y=66.26+5.74 X_{1}+2.09 X_{2}-0.49 X_{3}-0.23 X_{1} X_{2} \\
& -4.24 X_{1} X_{3}-0.41 X_{2} X_{3}-2.71 X_{1}^{2}-7.559 X_{2}^{2}-8.29 X_{3}^{2}
\end{aligned}
$$

Table 1: Independent variables and their levels in the response surface design

\begin{tabular}{llll}
\hline \multicolumn{3}{c}{ Levels } & \\
& ------------------ \\
Independent variables & -1 & 0 & 1 \\
\hline $\mathrm{X}_{1}$ Chelation time $(\mathrm{h})$ & 3 & 4 & 5 \\
$\mathrm{X}_{2}$ Chelation temperature $\left({ }^{\circ} \mathrm{C}\right)$ & 40 & 50 & 60 \\
$\mathrm{X}_{3} \mathrm{pH}$ & 4 & 5 & 6 \\
\hline
\end{tabular}

Table 3 showed the analysis of variance of the response surface quadratic model. The high $\mathrm{F}$ value (30.55) and low $\mathrm{P}$ value $(\mathrm{p}<0.01)$ confirmed the high validity of the regression equation and model. The regression equation and model could describe the chelation process of APP3a-Zn.

Linear $\left(\mathrm{X}_{1}\right.$ and $\left.\mathrm{X}_{2}\right)$ and quadratic coefficients $\left(\mathrm{X}_{1}{ }^{2}\right.$, $\mathrm{X}_{2}{ }^{2}$ and $\mathrm{X}_{3}{ }^{2}$ ) were significant for chelation rate $(p<0.05$ or $p<0.01)$. The independent variables (chelation time $\left(\mathrm{X}_{1}\right)$, chelation temperature $\left(\mathrm{X}_{2}\right)$ were the most important factors with a greater effect on chelation rate of APP3a $-\mathrm{Zn}$. 
Xinyi Wu et al. / American Journal of Biochemistry and Biotechnology 2021, 17 (1): 118.129 DOI: 10.3844/ajbbsp.2021.118.129

Table 2: Result of response surface analysis of the variation of chelation rate of APP3a-Zn with chelation time $\left(\mathrm{X}_{1}\right)$, chelation temperature $\left(\mathrm{X}_{2}\right)$ and $\mathrm{pH}\left(\mathrm{X}_{3}\right)$

\begin{tabular}{lllll}
\hline & Factors & & & \\
Runs & $-\mathrm{X}_{1}$ & $\mathrm{X}_{2}$ & \\
\hline 1 & -1 & 0 & $\mathrm{X}_{3}$ & Chelation rate $(\%)$ \\
2 & 1 & 1 & -1 & 44.2 \\
3 & 1 & 0 & 0 & 64.1 \\
4 & 0 & 1 & -1 & 65.4 \\
5 & 1 & 0 & -1 & 54.4 \\
6 & 0 & -1 & 1 & 57 \\
7 & 0 & 0 & 0 & 48 \\
8 & -1 & 1 & 0 & 67 \\
9 & 0 & 0 & 0 & 54.3 \\
10 & 0 & 0 & 0 & 67 \\
11 & 0 & -1 & -1 & 66.8 \\
12 & 1 & -1 & 0 & 49.2 \\
13 & -1 & -1 & 0 & 58.9 \\
14 & 0 & 0 & 0 & 68.2 \\
15 & 0 & 1 & 0 & 67.3 \\
16 & 0 & 0 & 0 & 57.4 \\
17 & -1 & 0 & 1 & 53.6 \\
\hline
\end{tabular}

Table 3: Analysis of variance for response surface quadratic model

\begin{tabular}{lccccc}
\hline Source & Sum of squares & Df & Mean squares & F-value & P-value \\
\hline Model & 905.59 & 9 & 100.62 & 30.550 & $<0.0001^{* *}$ \\
$\mathrm{X}_{1}$ & 283.58 & 1 & 283.58 & 86.110 & $<0.0001^{* *}$ \\
$\mathrm{X}_{2}$ & 29.71 & 1 & 29.71 & 9.020 & $0.0198^{*}$ \\
$\mathrm{X}_{3}$ & 1.49 & 1 & 1.49 & 0.450 & 0.5223 \\
$\mathrm{X}_{1} \mathrm{X}_{2}$ & 0.20 & 1 & 0.20 & 0.061 & 0.8113 \\
$\mathrm{X}_{1} \mathrm{X}_{3}$ & 83.23 & 1 & 83.23 & 25.270 & $0.0015^{* *}$ \\
$\mathrm{X}_{2} \mathrm{X}_{3}$ & 0.37 & 1 & 0.37 & 0.110 & 0.7479 \\
$\mathrm{X}_{1}^{2}$ & 26.99 & 1 & 26.99 & 6.200 & $0.0242^{*}$ \\
$\mathrm{X}_{2}{ }^{2}$ & 211.94 & 1 & 211.94 & 71.230 & $<0.0001^{* *}$ \\
$\mathrm{X}_{3}{ }^{2}$ & 234.6 & 7 & 234.60 & 17.100 & $<0.0001^{* *}$ \\
Residual & 23.05 & 3 & 3.29 & & $0.0096^{* *}$ \\
Lake of fit & 21.39 & 4 & 0.42 & & \\
Pure error & 1.67 & 16 & & & \\
Cor total & 928.64 & & &
\end{tabular}

Notes: * indicated significant difference $(p<0.05) ; * *$ indicated extremely significant difference $(p<0.01)$

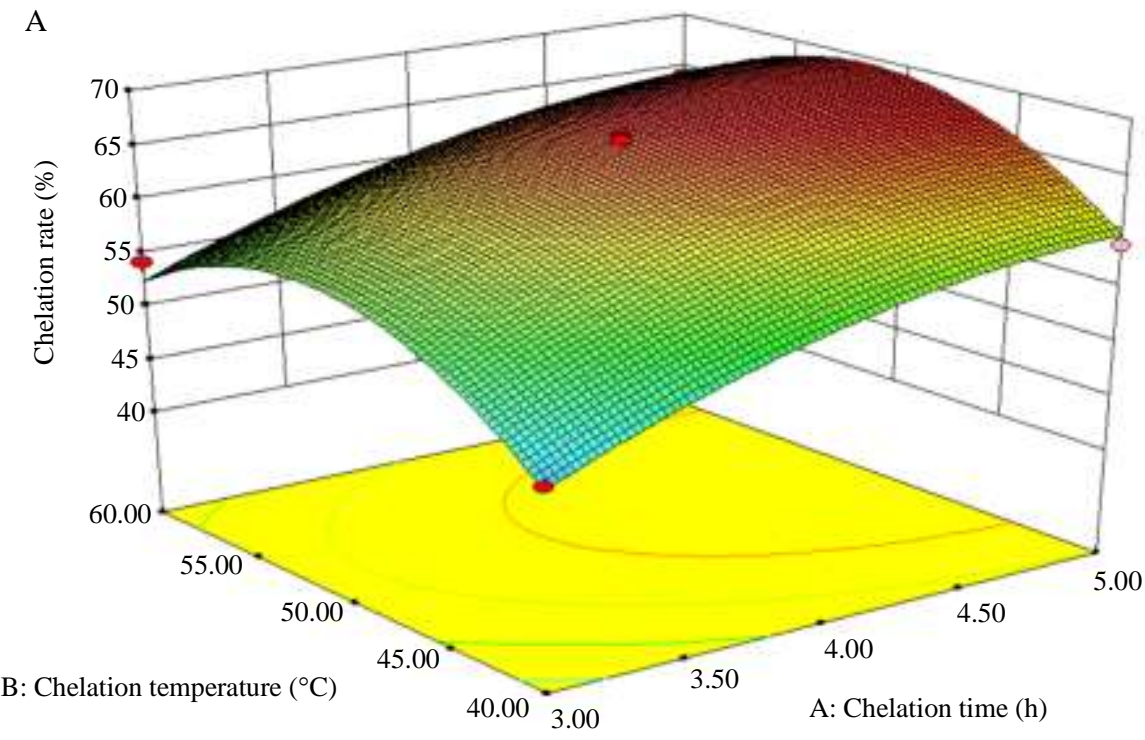



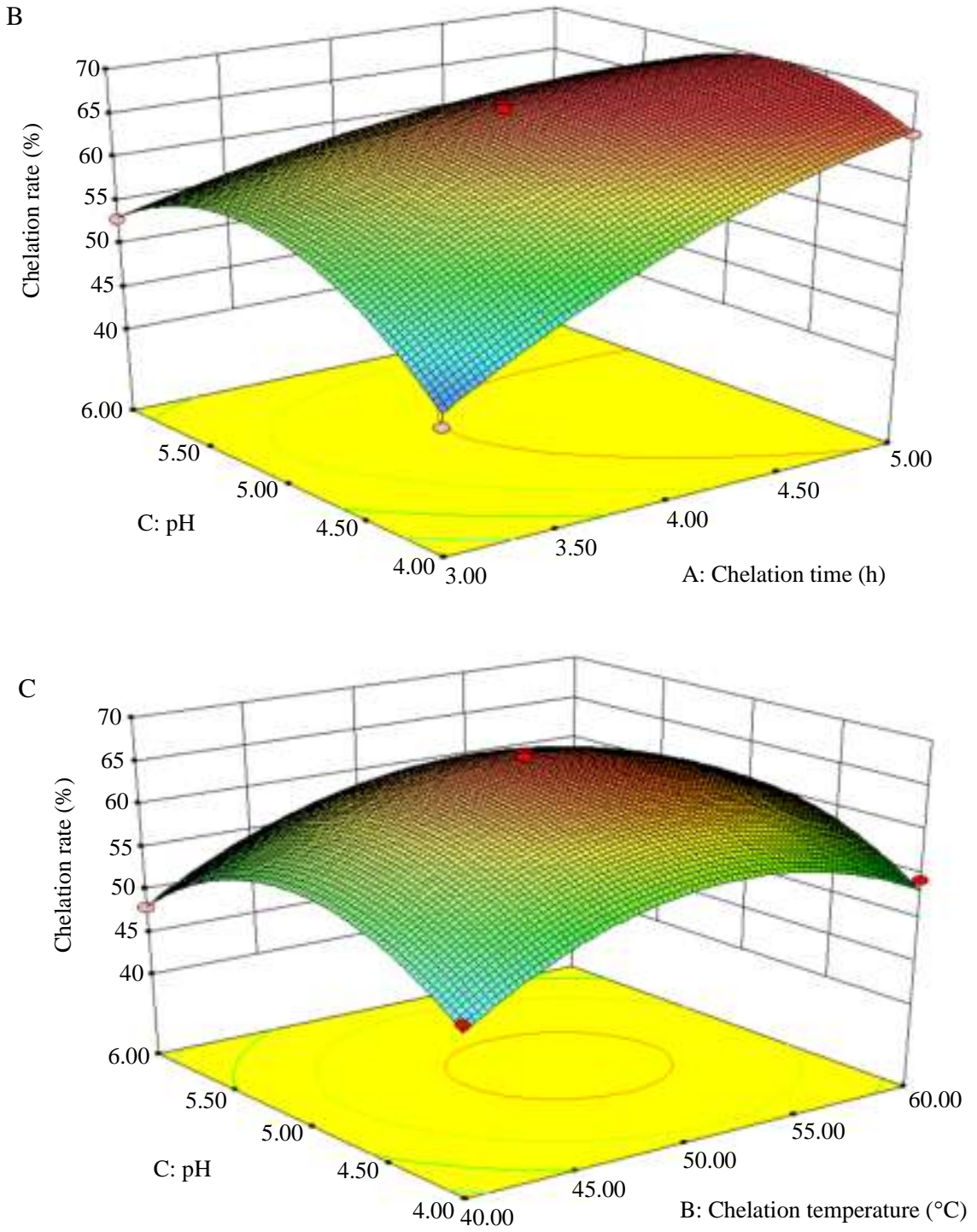

Fig. 2: Effect of chelation time and chelation temperature (A), chelation time and $\mathrm{pH}(\mathrm{B})$ and chelation temperature and $\mathrm{pH}(\mathrm{C})$ on chelation rate of APP3a- $\mathrm{Zn}$ in response surface plots

\section{Optimization of Preparation Process of APP3a $-\mathrm{Zn}$ and Validation of the Model}

The 3D response surface reflected the relationship between the two independent variables, as shown in Fig. 2.

Effect of chelation time and chelation temperature and their interactions on chelation rate of APP3a-Zn was seen in Fig. 2A. As the chelation temperature continued to increase, chelation rate also increased. When chelation temperature further increased, chelation rate began to decrease. Their interaction was not significant $(\mathrm{p}>0.05)$. When chelation time increased, chelation rate also increased. Compared with $\mathrm{pH}$, chelation time had a more significant effect on chelation rate of APP3a-Zn (Fig. 2B). Their interaction was extremely significant $(p<0.01)$.
When $\mathrm{pH}$ increased, chelation rate also increased. However, when $\mathrm{pH}$ further increased, chelation rate began to decrease (Fig. 2C). The interaction between $\mathrm{pH}$ and chelation temperature was not significant ( $p>0.05)$.

By analyzing the effect of chelating conditions on the chelating rate, the preparation process of APP3a-Zn was optimized. The best preparation process of APP3a- $\mathrm{Zn}$ was chelation time $4.45 \mathrm{~h}$, chelation temperature $49.37^{\circ} \mathrm{C}$ and $\mathrm{pH}$ 4.68. The maximum chelation rate of APP3a-Zn was $68.05 \%$.

To verify the adequacy of the model equation, three verification experiments were performed under conditions modified for convenience as following: Chelation time was $4.5 \mathrm{~h}$, chelation temperature was $50^{\circ} \mathrm{C}$ and $\mathrm{pH}$ was 4.7 . The average chelation rate of 
APP3a-Zn was $68.11 \%$, which was in good agreement with the predicted value $(68.06 \%)$. The result showed that the regression model was accurate and can predict the experimental result in practice.

\section{Zinc Content in APP3a-Zn}

Zinc content in APP3a-Zn and APP3a was shown in Table 4. No zinc was found in APP3a. However, after the combination of APP3a with zinc, the zinc content $(8.56 \pm 0.36 \%$, w/w) was significantly elevated in APP3a-Zn. The result also suggested that zinc was successfully in conjunction with APP3a.

\section{FT-IR Spectroscopy}

Infrared spectrum of APP3a-Zn and APP3a was seen in Fig. 3A. It could be seen that a broad peak around
$3400 \mathrm{~cm}^{-1}$ was the stretching vibration absorption peak of O-H group. The weak band around $2910 \mathrm{~cm}^{-1}$ was assigned to the vibration of $\mathrm{C}-\mathrm{H}$ group. Compared with the FT-IR spectrum of APP3a, a weak peak of APP3a-Zn appeared at $1640 \mathrm{~cm}^{-1}$ may be due to the tensile vibration of $\mathrm{Zn}-\mathrm{O}$, resulting in the change of hydrogen bonding between $\mathrm{OH}$ groups. Crosslinking may be due to electrostatic attraction between the positive charge of zinc ions and the negative charge of polysaccharides (Wang and $\mathrm{Li}, 2019$ ).

Table 4: Results of determination of zinc content in APP3a-Zn and APP3a

\begin{tabular}{ll}
\hline Sample & Zinc content $(\%)$ \\
\hline APP3a & 0 \\
APP3a-Zn & $8.56 \pm 0.36$
\end{tabular}
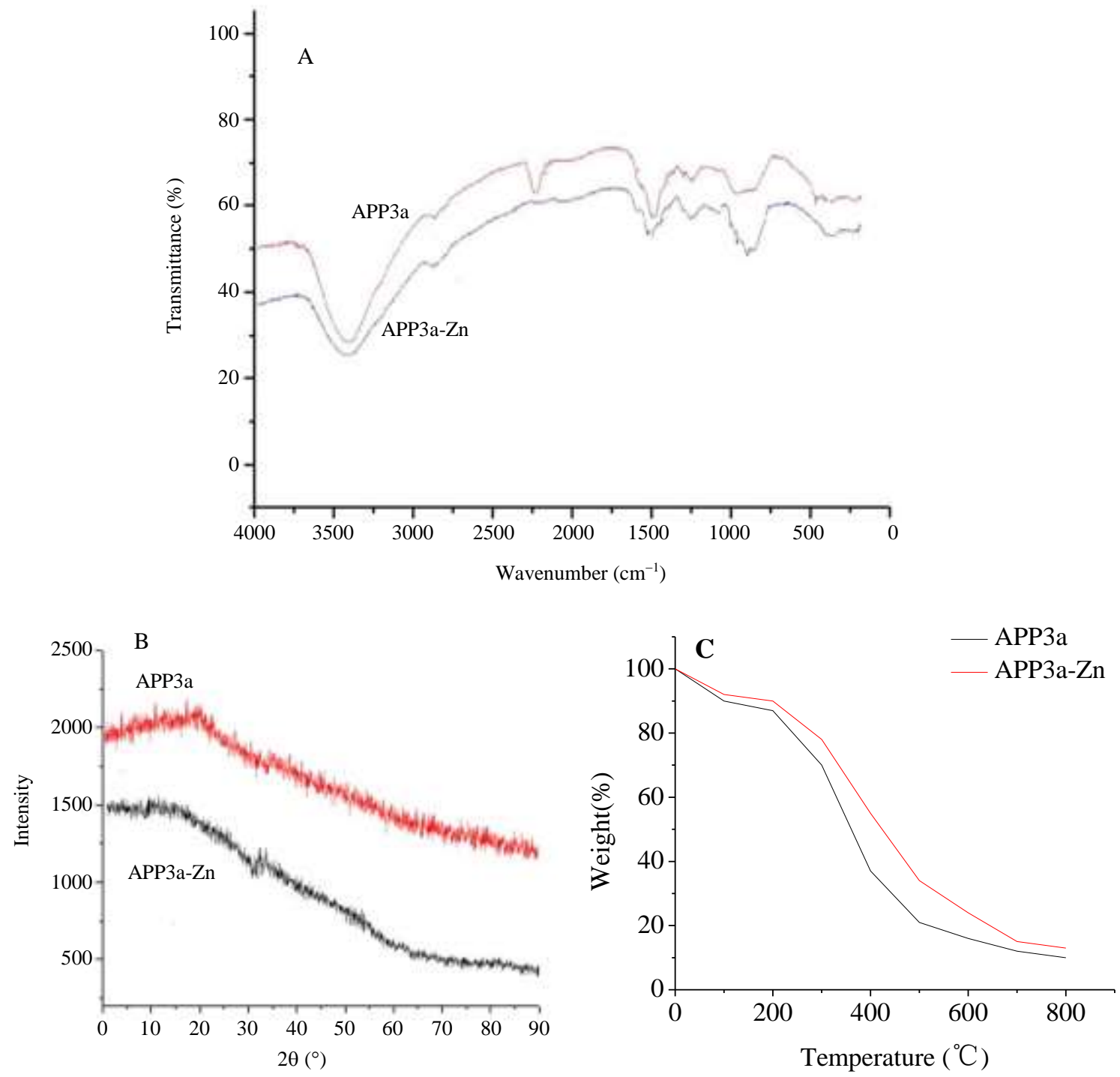

Fig. 3: FT-IR spectroscopy analysis (A), X-ray diffraction spectrum (B) and thermogravimetric curve (C) of APP3a-Zn and APP3a 

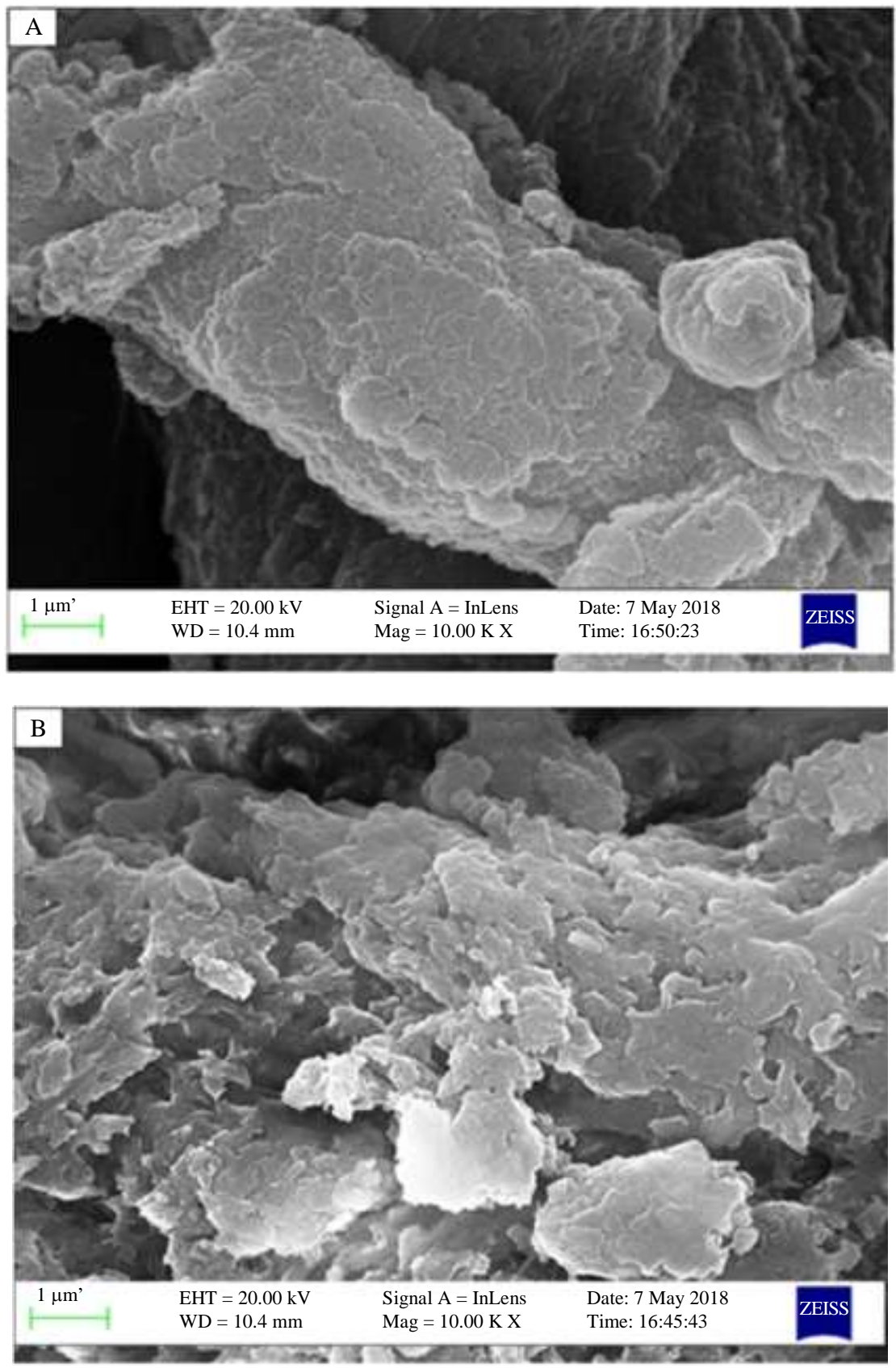

Fig. 4: Scanning electron microscope image of APP3a (A) and APP3a-Zn (B) at 10000x

\section{X-Ray Diffraction Spectrum}

$\mathrm{X}$-ray diffraction spectrum profile of APP3a and APP3a-Zn was shown in Fig. 3B. It was observed that APP3a and APP3a-Zn had no high crystallinity and sharp crystal peaks. There was a "bun-shaped" peak in APP3a without sharp peaks, which showed that APP3a tended to be a low total crystallinity substance. After chelation with zinc, the broad peak of APP3a disappeared, indicating that the crystal structure of APP3a- $\mathrm{Zn}$ had undergone tremendous changes.

\section{Thermogravimetric Analysis}

Thermogravimetric curve of APP3a and APP3a-Zn was presented in Fig. 3C. The weight loss of APP3a and APP3a-Zn involved in three steps. First of all, the weight of APP3a-Zn had a tendency to decline slowly within the range of $200^{\circ} \mathrm{C}$ like that of APP3a because of the loss of physically bound water. Secondly, the weight loss trend dropped sharply from 200 to $600^{\circ} \mathrm{C}$ because of the loss of chemically bound water caused by oxidation. Finally, when the temperature exceeded 
$600^{\circ} \mathrm{C}$, relatively little change in weight loss was observed. Compared with APP3a-Zn, the weight loss of APP3a was similar with that of APP3a-Zn. However, the weight loss of APP3a-Zn was slightly smaller than that of APP3a within the heating temperature range. The reason might be that the newly introduced $\mathrm{Zn}-\mathrm{O}$ group replaced the $\mathrm{H}-\mathrm{O}$ group in APP3a and changed the structural characteristics, which resulted in the reduction of physical and chemical water.

\section{Morphological Analysis}

The morphological structures of APP3a-Zn and APP3a was analyzed and compared with SEM. The SEM images of both shown in Fig. 4 were clearly different. The surface of APP3a was relatively smooth and flat (Fig. 4A). After chelating with Zn, APP3a-Zn was hairy and the surface was rough (Fig. 4B). The reason might be that the electric charge was neutralized after combining with zinc and the spatial conformation of APP3a tended to assemble.

\section{Antioxidant Activities of APP3a and APP3a-Zn}

In the present study, the antioxidant activity of APP3a and APP3a-Zn by scavenging DPPH free radicals, hydroxyl free radicals and superoxide anion free radicals was investigated. Antioxidant activities of APP3a and APP3a-Zn were presented in Fig. 5.

As shown in Fig. 5A, with the increase of concentration of APP3a and APP3a-Zn, the scavenging ability of hydroxyl radical increased. The scavenging ability of APP3a-Zn was stronger than that of APP3a.

As shown in Fig. 5B, APP3a and APP3a-Zn had a significant effect on scavenging DPPH free radicals with a concentration-dependent manner. The scavenging capacity of APP3a-Zn on DPPH radical was higher than that of APP3a.
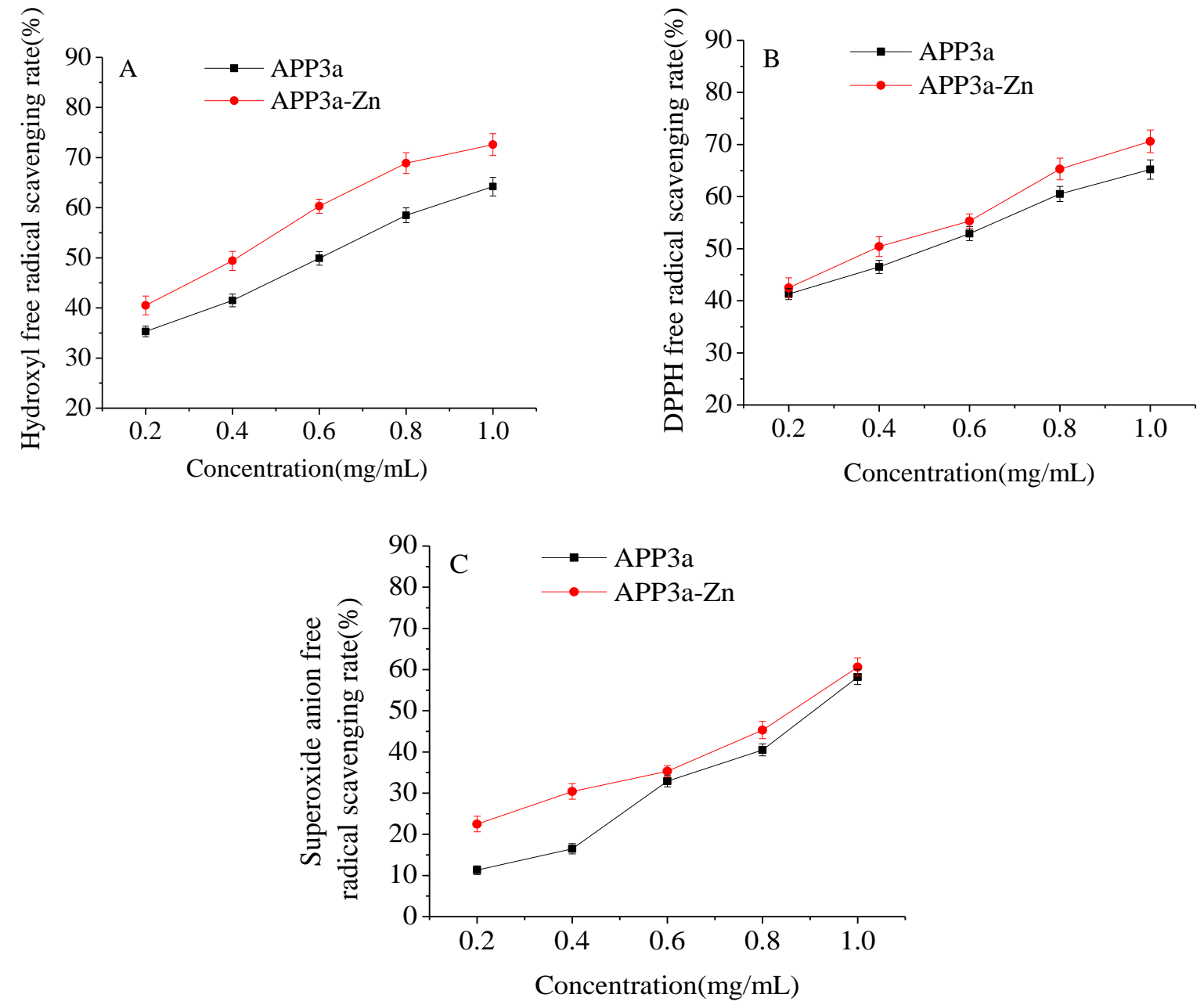

Fig. 5: Scavenging Effect of APP3a and APP3a-Zn on hydroxyl free radical (A), DPPH free radical (B) and superoxide anion free radical $(C)$ 
As shown in Fig. 5C. APP3a and APP3a-Zn had the obvious scavenging effect on superoxide anion free radicals with a concentration-dependent manner. The scavenging capacity of APP3a-Zn on superoxide anion free radicals was higher than that of APP3a. The results not only confirmed that the antioxidant activity of APP3a$\mathrm{Zn}$, but also elucidated that after the chelation of APP3a and zinc, the antioxidant activity of APP3a-Zn did not decrease, but increased further.

\section{Discussion}

Recently, people are paying more and more attention to zinc-rich polysaccharides that have higher antioxidant activity than conventional polysaccharides. To the best of our knowledge, there is no report on optimization of preparation technology, characterization and biological activity of $A$. polytricha polysaccharide-Zinc(II) complex.

As an important indicator of antioxidant activity, ROS is commonly used to assess the health benefits of bioactive polysaccharides. In fact, ROS as free radicals includes hydroxyl radical, superoxide anion radical and non-radical substances (Tohma et al., 2017). Hydroxyl radical is one of the most harmful free radicals, which can cause oxidative damage and cell necrosis or mutation through addition, dehydrogenation and electron transfer $(\mathrm{Xu}$ et al., 2019). DPPH is a stable nitrogen-centered organic free radical, widely used in the screening of natural antioxidants (Hung et al., 2006). The aging process from skin to internal organs can be accelerated by superoxide anion free radicals, which induces cardiovascular diseases, skin diseases and cancer (Lagercrantz, 1999). In the present study, the scavenging ability of APP3a-Zn on hydroxyl radical, DPPH radical and superoxide anion free radicals was stronger than that of APP3a. It might be because the coordination of APP3a and $\mathrm{Zn}$ reduced the exposed active groups and weakened its combination with hydroxyl radicals.

Changes in the spatial conformation of the APP3a molecule including the number of zinc binding sites and the binding strength in the complex resulted in decrease of antioxidant activity of App3a. APP3a-Zn and superoxide anion free radicals formed a more stable intermediate, which resulted in increasing the ability to scavenge superoxide anion free radicals (Lagercrantz, 1999).

\section{Conclusion}

In our previous work, a novel polysaccharide APP3a was obtained. The preparation process of APP3a-Zn was optimized by RSM. Characterization of APP3a-Zn was carried out and its antioxidant activity was also investigated. The best preparation process of APP3a-Zn was chelation time $4.45 \mathrm{~h}$, chelation temperature $49.37^{\circ} \mathrm{C}$ and $\mathrm{pH} 4.68$. The weight loss of APP3a-Zn was slightly smaller than that of APP3a within the heating temperature range and the crystal structure of APP3a-Zn had undergone tremendous changes compared with APP3a. APP3a-Zn had stronger antioxidant activity than APP3a, which indicated that chelation with zinc did not change the antioxidant activity of APP3a, but enhanced the antioxidant activity of APP3a. In the food industry, APP3a-Zn had great potential as a new zinc supplement with antioxidant activity. Meanwhile, the present study might provide a new way to develop new zinc supplement for human.

\section{Acknowledgement}

This research was supported by college student's innovation and entrepreneurship training program project of Jiangsu provincial colleges $(202010333076 \mathrm{H})$.

\section{Author's Contributions}

Xinyi Wu and Xiao Ding: Participated in all experiments and article writing.

Ze Zhang and Ziyi Xu: Participated in characterization and optimization of preparation process of APP3a-Zn and data processing.

Jiehan Zhang: Participated in antioxidant activities of APP3a-Zn.

Wen Zhao: Responsible for data analysis.

Yiyong Chen: Participated in project design and experimental guidance of this manuscript.

\section{Ethics}

This article is original and contains unpublished material. The corresponding author confirms that all of the other authors have read and approved the manuscript and no ethical issues involved.

\section{References}

Beyersmann, D., \& Haase, H. (2001). Functions of zinc in signaling, proliferation and differentiation of mammalian cells. Biometals, 14(3), 331-341. https://doi.org/10.1023/A:1012905406548

Boroujeni, S. T., Naghdi, N., Shahbazi, M., Farrokhi, A., Bagherzadeh, F., Kazemnejad, A., \& Javadian, M. (2009). The effect of severe zinc deficiency and zinc supplement on spatial learning and memory. Biological Trace Element Research, 130(1), 48-61. https://doi.org/10.1007/s12011-008-8312-7

Buff, C. E., Bollinger, D. W., Ellersieck, M. R., Brommelsiek, W. A., \& Veum, T. L. (2005). Comparison of growth performance and zinc absorption, retention and excretion in weanling pigs fed diets supplemented with zinc-polysaccharide or zinc oxide. Journal of Animal Science, 83(10), 2380-2386. https://doi.org/10.2527/2005.83102380x 
Caulfield, L. E., de Onis, M., Blössner, M., \& Black, R. E. (2004a). Undernutrition as an underlying cause of child deaths associated with diarrhea, pneumonia, malaria and measles. The American Journal of Clinical Nutrition, 80(1), 193-198. https://doi.org/10.1093/ajcn/80.1.193

Caulfield, L. E., Richard, S. A., \& Black, R. E. (2004b). Undernutrition as an underlying cause of malaria morbidity and mortality in children less than five years old. The American Journal of Tropical Medicine and Hygiene, 71(2_suppl), 55-63. https://doi.org/10.4269/ajtmh.2004.71.55

Chen, Y., \& Xue, Y. (2018). Purification, chemical characterization and antioxidant activities of a novel polysaccharide from Auricularia polytricha. International Journal of Biological Macromolecules, 120 , 1087-1092. https://doi.org/10.1016/j.ijbiomac.2018.08.160

Harmaza, Y. M., \& Slobozhanina, E. I. (2014). Zinc essentiality and toxicity. Biophysical Aspects. Biophysics, 59(2), 264-275 https://doi.org/10.1134/S0006350914020092

Hung, T. M., Na, M., Thuong, P. T., Su, N. D., Sok, D., Song, K. S., ... \& Bae, K. (2006). Antioxidant activity of caffeoyl quinic acid derivatives from the roots of Dipsacus asper Wall. Journal of ethnopharmacology, 108(2), 188-192. https://doi.org/10.1016/j.jep.2006.04.029

Imai, M., Tanaka, M., Tanaka, K., Yamamoto, Y., ImaiOgata, N., Shimowatari, M., ... \& Suemune, H. (2004). Double-chelation-assisted Rh-catalyzed intermolecular hydroacylation between salicylaldehydes and 1, 4-penta-or 1, 5-hexadienes. The Journal of Organic Chemistry, 69(4), 1144-1150. https://doi.org/10.1021/jo035395u

Lagercrantz, C. (1999). Formation of the paramagnetic complex $[\mathrm{Cr}(\mathrm{OH}) 5 \mathrm{O} 2] 5-$ in the reaction between chromium (VI) oxide and hydrogen peroxide or superoxide anion radicals studied by EPR spectroscopy. Free Radical Biology and Medicine, 26(9-10), 1134-1137. https://doi.org/10.1016/S0891-5849(98)00294-9

Li, X., Hou, R., Yue, C., Liu, J., Gao, Z., Chen, J., ... \& $\mathrm{Hu}, \mathrm{Y}$. (2016). The selenylation modification of Epimedium polysaccharide and Isatis root polysaccharide and the immune-enhancing activity comparison of their modifiers. Biological Trace Element Research, 171(1), 224-234. https://doi.org/10.1007/s12011-015-0511-4

Lian, K. X., Zhu, X. Q., Chen, J., Liu, G., \& Gu, X. L. (2018). Selenylation modification: Enhancement of the antioxidant activity of a Glycyrrhiza uralensis polysaccharide. Glycoconjugate Journal, 35(2), 243-253. https://doi.org/10.1007/s10719-018-9817-8
Sheu, F., Chien, P. J., Chien, A. L., Chen, Y. F., \& Chin, K. L. (2004). Isolation and characterization of an immunomodulatory protein (APP) from the Jew's Ear mushroom Auricularia polytricha. Food Chemistry, 87(4), 593-600. https://doi.org/10.1016/j.foodchem.2004.01.015

Tohma, H., Gülçin, İ., Bursal, E., Gören, A. C., Alwasel, S. H., \& Köksal, E. (2017). Antioxidant activity and phenolic compounds of ginger (Zingiber officinale Rosc.) determined by HPLC-MS/MS. Journal of Food Measurement and Characterization, 11(2), 556-566. https://doi.org/10.1007/s11694-016-9423-z

Wang, H., \& Betti, M. (2017). Sulfated glycosaminoglycan-derived oligosaccharides produced from chicken connective tissue promote iron uptake in a human intestinal Caco-2 cell line. Food Chemistry, 220, 460-469. https://doi.org/10.1016/j.foodchem.2016.10.025

Wang, L., \& Li, X. (2019). Preparation, physicochemical property and in vitro antioxidant activity of zincHohenbuehelia serotina polysaccharides complex. International journal of Biological Macromolecules, 121 862-869. https://doi.org/10.1016/j.ijbiomac.2018.10.118

Wang, Z. J., Xie, J. H., Shen, M. Y., Tang, W., Wang, H., Nie, S. P., \& Xie, M. Y. (2016). Carboxymethylation of polysaccharide from Cyclocarya paliurus and their characterization and antioxidant properties evaluation. Carbohydrate Polymers, 136, 988-994. https://doi.org/10.1016/j.carbpol.2015.10.017

Wang, L., Song, S., Zhang, B., Ai, C., Wen, C., Gong, Y., ... \& Xiao, H. (2019b). A sulfated polysaccharide from abalone influences iron uptake by the contrary impacts of its chelating and reducing activities. International Journal of Biological Macromolecules, 138, 49-56. https://doi.org/10.1016/j.ijbiomac.2019.07.072

Wang, P. P., Huang, Q., Chen, C., You, L. J., Liu, R. H., Luo, Z. G., ... \& Fu, X. (2019a). The chemical structure and biological activities of a novel polysaccharide obtained from Fructus Mori and its zinc derivative. Journal of Functional Foods, 54, 64-73. https://doi.org/10.1016/j.jff.2019.01.008

Xu, L., Tsona, N. T., Tang, S., Li, J., \& Du, L. (2019). Role of $(\mathrm{H} 2 \mathrm{O}) \mathrm{n}(\mathrm{n}=1-2)$ in the gas-phase reaction of ethanol with hydroxyl radical: mechanism, kinetics and products. ACS Omega, 4(3), 5805-5817. https://doi.org/10.1021/acsomega.9b00145

Yu, J., Sun, R., Zhao, Z., \& Wang, Y. (2014). Auricularia polytricha polysaccharides induce cell cycle arrest and apoptosis in human lung cancer A549 cells. International Journal of Biological Macromolecules, 68, 67-71. https://doi.org/10.1016/j.ijbiomac.2014.04.018 
Xinyi Wu et al. / American Journal of Biochemistry and Biotechnology 2021, 17 (1): 118.129 DOI: 10.3844/ajbbsp.2021.118.129

Zhao, S., Rong, C., Liu, Y., Xu, F., Wang, S., Duan, C., .. \& Wu, X. (2015). Extraction of a soluble polysaccharide from Auricularia polytricha and evaluation of its anti-hypercholesterolemic effect in rats. Carbohydrate Polymers, 122, 39-45. https://doi.org/10.1016/j.carbpol.2014.12.041
Zhao, S., Li, B., Chen, G., Hu, Q., \& Zhao, L. (2017). Preparation, characterization and anti-inflammatory effect of the chelate of Flammulina velutipes polysaccharide with $\mathrm{Zn}$. Food and Agricultural Immunology, 28(1), 162-177. https://doi.org/10.1080/09540105.2016.1230600 